\title{
Novel topical therapy for mild-to-moderate plaque psoriasis: focus on calcitriol
}

This article was published in the following Dove Press journal:

Clinical, Cosmetic and Investigational Dermatology

15 September 2009

Number of times this article has been viewed

\author{
Lutz Kowalzick \\ Department of Dermatology \\ and Allergology, HELIOS \\ Vogtland-Klinikum Plauen GmbH \\ Plauen, Germany
}

Correspondence: Lutz Kowalzick

Klinik für Hautkrankheiten und

Allergologie, HELIOS Vogtland-Klinikum

Plauen GmbH, Box I00 I53, D-08505

Plauen, Germany

$\mathrm{Tel}+44$ 374I 4924I0

Fax +44 374I 4924II

Email lutz.kowalzick@helios-kliniken.de

\begin{abstract}
The benefits of vitamin D derivatives for the treatment of chronic plaque psoriasis are well documented. Of importance is how compatible they are with, and how they compare to, other established treatments for psoriasis. This paper reviews 15 studies with calcitriol $3 \mu \mathrm{g} \mathrm{g}^{-1}$ ointment (Silkis ${ }^{\circledR} /$ Vectical $^{\mathrm{TM}}$ ointment; Galderma Laboratories) applied twice daily for up to 52 weeks. The ointment was found to be effective and safe for the treatment of mild-to-moderate plaque psoriasis including sensitive skin areas. Calcitriol $3 \mu \mathrm{g} \mathrm{g}^{-1}$ ointment was found to be as effective as, or even superior to, other established treatment modalities and more highly tolerated than other local treatment modalities in most studies' comparisons. It could be combined with other psoriasis treatment modalities such as ultraviolet B phototherapy or topical corticosteroids in order to achieve a faster response and in order to reduce the risk of adverse events.
\end{abstract}

Keywords: 1,25-dihydroxyvitamin $\mathrm{D}_{3}$, calcitriol, psoriasis, therapy

\section{Introduction}

Psoriasis is a chronic inflammatory disease of the skin which affects about $2 \%$ to $3 \%$ of the population. About $15 \%$ to $20 \%$ of these patients suffer from additional inflammatory diseases of their joints. Some authors state that psoriatic arthritis is more common in severe and advanced cases of skin psoriasis, but in up to $15 \%$ of cases it precedes the skin disease and about 6\% never develop skin lesions. Psoriasis of finger or toe nail involvement is also observed in many cases. The most frequent clinical form of the skin disease is plaque psoriasis with lesions preferentially located on the scalp, elbows, knees and the sacral area. Established topical treatments of plaque psoriasis include salicylic acid, corticosteroids, dithranol, tar, and both vitamin A and D derivatives. In cases recalcitrant to topical therapy or extensive or with palmoplantar skin area involvement additional ultraviolet phototherapy or, alternatively, systemic treatment modalities including methotrexate, retinoids, cyclosporine, fumarates and biologics could be administered.

The overall effectiveness of topical vitamin $\mathrm{D}_{3}$ derivatives in treating psoriasis is well documented. Generally, patients show good tolerance to these agents, with no marked disturbance in their calcium metabolism, ${ }^{1,2}$ although hypercalcemia does occur occasionally ${ }^{3,4}$ and some patients reported skin irritations ${ }^{5,6}$ The important consideration for vitamin D derivatives now is their comparability and compatibility, both in terms of effectiveness and tolerance, with other established treatments for psoriasis.

An ointment with $3 \mu \mathrm{g} \mathrm{g}^{-1}$ calcitriol (Silkis ${ }^{\circledR} /$ Vectical $^{\mathrm{TM}}$ ointment; Galderma Laboratories) was approved in Europe about 10 years ago and recently in the US for the treatment of mild-to-moderate plaque psoriasis. This paper reviews a 
number of studies in order to explore the characteristics of calcitriol and to establish its place in the armamentarium available for the management of patients with psoriasis.

\section{Pharmacology, mode of action, and pharmacokinetics}

The role of calcitriol (1,25-dihydroxyvitamin $\left.\mathrm{D}_{3}\right)$, a naturally occurring endogenous hormonally-active derivative of vitamin $\mathrm{D}$, in keratinocyte proliferation and differentiation, together with its effects on the immune response, are the reasons for the interest in its potential to control hyperproliferative, inflammatory skin diseases such as psoriasis. Receptors for calcitriol are found on epidermal keratinocytes and dermal fibroblasts. ${ }^{7}$ Furthermore, keratinocyte growth and differentiation are regulated by calcium concentration, ${ }^{8}$ and as calcitriol increases intracellular calcium concentration, ${ }^{9}$ these processes may be indirectly affected by calcitriol. In vitro physiological studies have revealed that calcitriol inhibits the proliferation of human skin cells and induces them to differentiate into squamous and denucleated horny cells. ${ }^{10,11}$ Additionally, calcitriol enhances the expression of keratins $\mathrm{K} 10$ and $\mathrm{K} 6$ in the keratinocyte population in psoriasis plaques in vivo, which correlates with their clinical improvement. ${ }^{12}$ Furthermore, it has been shown that calcitriol acts as an immunomodulator in the skin as a potent inhibitor of T-cell proliferation and of several inflammatory mediators including interleukins-1, -6 , and $-8 .{ }^{13,14}$

In preclinical and phase I to III studies it was demonstrated that $3 \mu \mathrm{g} \mathrm{g}^{-1}$ calcitriol ointment is the minimal optimal effective dose for treatment of psoriasis, had no systemic toxicity and was tolerated well in the locality of application. ${ }^{15}$

In humans, following topical administration of $3 \mu \mathrm{g} \mathrm{g}^{-1}$ calcitriol ointment to about $15 \%$ of the body surface area for 12 hours, on average $7.5 \%$ of the calcitriol applied was excreted mainly via feces over a 10-day period. The level of serum calcitriol remained unchanged and traces of exogenous calcitriol were only detected in $25 \%$ of participants in the relevant study. ${ }^{15}$ The main concern about the use of vitamin $\mathrm{D}$ derivatives is their possible influence on calcium homeostasis. In animals treated with $3 \mu \mathrm{g} \mathrm{g}^{-1}$ calcitriol ointment (on $15 \%$ of the body surface for four consecutive days) no increase in the urine calcium excretion could be observed as compared to animals treated with the excipient over 10 days. ${ }^{16}$ For health and safety reasons, in Europe, the use of $3 \mu \mathrm{g} \mathrm{g}^{-1}$ calcitriol ointment is only approved for treatment of up to $35 \%$ of the body surface area in psoriasis patients with a maximum of $30 \mathrm{~g}$ per day being the approved level in the US. No irritant, sensitizing, phototoxic or photoallergic potential of $3 \mu \mathrm{g} \mathrm{g} \mathrm{g}^{-1}$ calcitriol ointment could be detected in animal and human phase I studies. ${ }^{15}$

\section{Clinical studies - general methodology}

Several separate studies designed to asses the efficacy, tolerability, and safety of applying calcitriol $3 \mu \mathrm{g} \mathrm{g}^{-1}$ ointment twice a day are reviewed here; two were comparisons with an excipient, two were comparisons with other standard treatments for psoriasis, two were comparisons with calcipotriol, another vitamin $\mathrm{D}$ derivative, one was a comparison with tacrolimus, a drug not yet approved for psoriasis, four investigated the combination of calcitriol with other therapies, and four were open, uncontrolled investigations. Most of these studies have been reported in detail elsewhere. ${ }^{17-28}$

All studies were conducted with adult patients of both sexes with chronic plaque-type psoriasis. Unless otherwise specified, treatments were applied to all psoriatic lesions (except the head) for the duration of the study or until the lesions cleared up. Efficacy was mostly assessed in terms of global improvement, with erythema, scaling, induration and pruritus being scored according to the scale of Fredriksson and colleagues ${ }^{29}$ or global severity scores (GSS), physician's global assessment scores (PGA), and dermatological sum scores (DSS). In many studies the psoriasis area and severity index (PASI) score was used as a secondary efficacy variable. ${ }^{30}$ In studies directed to treatment of special sensitive skin areas modified PASI or target area scores (TAS) were employed. Some studies included an evaluation of patients' quality of life during treatment using the psoriasis disability index (PDI) or quality of life (QOL) questionnaires about daily activities. ${ }^{31,32}$

Evaluations were performed at the beginning of each study, at regular intervals throughout the relevant study, and at the end of each study. Blood chemistry parameters, including serum albumin, total and adjusted calcium, urea, phosphate, alkaline phosphatase, and creatinine were also measured at assessment points in most studies.

\section{Efficacy, safety, and patient acceptance Comparison with excipient}

In two placebo-controlled multicenter randomized doubleblind parallel group studies including 839 subjects, $3 \mu \mathrm{g} \mathrm{g}^{-1}$ calcitriol ointment or its excipient was administered twice 
daily for up to eight weeks. In both studies the drug was shown to be significantly more effective $(P=0.005$ and $P<0.001$, respectively) according to the response rate, and both according to GSS and DSS $(P<0.01$ to $P<0.001$, respectively) in mild-to-moderate plaque psoriasis than its excipient. Furthermore, itching was significantly reduced in the calcitriol-treated group in both studies $(P<0.001)$. In both studies calcitriol ointment had no effect on calcium homeostasis as well demonstrating a good systemic and local safety profile in comparison to the groups being treated with the excipient. There was a higher occurrence of local adverse events in the relevant locality where the excipient was applied..$^{20}$

\section{Comparison with short-contact dithranol}

In an open, parallel-group study patients were randomized to eight weeks' treatment with either calcitriol $3 \mu \mathrm{g} \mathrm{g}^{-1}$ ointment applied twice daily or dithranol cream applied once daily. Dithranol cream had to be removed after $30 \mathrm{~min}$ by shower bath. Its concentration started at $0.25 \%$ for the first seven days, and then increased to $0 \%, 1 \%$, and $2 \%$ at seven-day intervals according to local tolerance. The global improvement scores and PASI scores were very similar between the two treatments; considerable or definite improvement, or total clearance of lesions, was observed in $72 \%$ of 60 calcitriol-treated patients and $70 \%$ of 54 dithranol-treated patients, respectively. At the end of the study the improvements measured against the start of the study in the PASI scores were 64\% and 57\%, respectively. In addition to standard assessments, the effect of psoriasis on the patients' quality of life was measured using the PDI questionnaire about daily activities. Patients were also asked to assess the acceptability of the two medications in terms of staining, smell, ease of spreading and irritation. Patients in the calcitriol group experienced a significantly higher quality of life during the study period, than those in the dithranol group $(P<0.05)$ as determined using the PDI questionnaire. There was also a significant difference in favor of calcitriol ointment in the rating of staining and irritation $(P<0.01)$ and a higher proportion in the calcitriol group rated the overall acceptability of treatment as good (47\%) than in the dithranol group $(20 \%){ }^{18}$

\section{Comparison with corticosteroid}

In a randomized multicenter trial with 258 patients with chronic plaque psoriasis either calcitriol $3 \mu \mathrm{g} \mathrm{g}^{-1}$ ointment or the topical corticosteroid betamethasone dipropionate were administered twice daily for six weeks. Global improvement and global severity scores at treatment endpoint showed statistically significant $(P<0.05)$ differences in favor of betamethasone dipropionate, however the absolute reduction in mean PASI was comparable between the groups. A significantly $(P<0.01)$ higher proportion of patients who responded remained in remission during the eight-week observation period following the calcitriol treatment $(48 \%)$ as compared to the betamethasone treatment $(25 \%)$. Seven patients in each treatment group reported local skin irritations. Two patients treated with calcitriol exhibited slight, transient asymptomatic hypercalcemia, whereas this observation was made in three cases of the betamethasone treated group. Patients' overall opinion of both ointments was generally "good" or "acceptable" in $91 \%$ of the calcitriol and in $92 \%$ of the betamethasone group, respectively. ${ }^{22}$

\section{Comparison with calcipotriol}

To compare the efficacy and safety of calcitriol $3 \mu \mathrm{g} \mathrm{g}^{-1}$ ointment and calcipotriol $50 \mu \mathrm{g} \mathrm{g}^{-1}$ ointment both were applied twice a day for 12 weeks, a multicenter, randomized investigator-blind parallel group study including 250 patients with mild-to-moderate plaque type psoriasis was performed. At endpoint the mean score of global improvement $(0=$ no change or worsening, 3 = cleared or almost cleared) rated by the physician was 2.27 for calcitriol and 2.22 for calcipitriol; such differences being statistically insignificant. The same parameter scored by the patients was 2.12 for calcitriol and 2.09 for calcipotriol; again showing statistically insignificant differences. Mild to moderate skin irritation was observed in percent of the patients treated with calcitriol and in $11 \%$ treated with calcipotriol. The mean worst score for the cutaneous safety assessment $(0=$ none, $4=$ very severe $)$ was lower in the calcitriol group (0.1) compared to the calcipotriol group (0.3) according to the physician's judgment. This difference was significant $(P<0.005)$ in favor of a better cutaneous safety profile for calcitriol. According to the patients' judgment, the mean worst score for the cutaneous discomfort was lower in the calcitriol group (0.2) compared to the calcipotriol group (0.4). This difference was significant $(P<0.05)$ in favor of a better acceptance for calcitriol. ${ }^{24}$

In another intra-individual, randomized, investigatorblinded left-right comparison study including 75 patients with symmetrical mild-to-moderate plaque psoriasis affecting sensitive areas (face, hairline, retroauricular and flexural areas) calcitriol $3 \mu \mathrm{g} \mathrm{g}^{-1}$ ointment was compared to calcipotriol $50 \mu \mathrm{g} \mathrm{g}^{-1}$ ointment both administered twice daily. Global assessment of improvements as compared to the beginning of the study by the investigators was significantly 
better for the calcitriol treated lesions and the subjects' global preference was in favor of calcitriol (both $P<0.05$ ). Perilesional erythema, edema and stinging or burning were statistically significantly ( $P<0.05$ to 0.001$)$ less severe with calcitriol compared to calcipotriol. The patients' evaluation of local tolerability in this study was significantly $(P<0.0001)$ in favor of calcitriol, whereas there was no patient preference regarding both treatments according to efficacy. ${ }^{25}$

\section{Comparison with tacrolimus}

To compare the efficacy of calcitriol $3 \mu \mathrm{g} \mathrm{g}^{-1}$ ointment and of the calcineurin antagonist tacrolimus in a $300 \mu \mathrm{g} \mathrm{g}^{-1}$ ointment either given twice daily for six weeks, a double-blind parallel group study was performed including 50 patients with chronic plaque psoriasis involving facial and genitofemoral areas. At the end of the study significantly $(P<0.05)$ more patients treated with tacrolimus $(60 \%)$ achieved complete or almost complete clearance according physicians global assessment compared to calcitriol (33\%). The same was true for a target area score which at the end of treatment decreased by $52 \%$ with calcitriol and $64 \%$ with tacrolimus, respectively. After two weeks, both calcitriol (71\%) and tacrolimus (48\%) induced considerable rates (nonsignificant) of perilesional erythema. After six weeks, calcitriol induced a statistically significant $(P<0.005)$ higher rate $(58 \%)$ compared to tacrolimus (16\%). Nonsignificant differences were found for edema, stinging/burning, and hot sensations, which occurred in very few cases. In regard to overall tolerance, both treatments were judged as excellent by $92 \%$ of the patients. ${ }^{27}$

\section{Combinations with ultraviolet $B$ phototherapy}

The efficacy of calcitriol $3 \mu \mathrm{g} \mathrm{g}^{-1}$ ointment in combination with ultraviolet (UV) B phototherapy was compared with phototherapy alone in an eight-week study. ${ }^{19}$ The research was a double-blind study done through the use of vehicle ointment in the control group. Patients received three sessions per week of broad-band UVB phototherapy until endpoint; dosage followed a defined schedule depending on the patient's skin type. The dose of UVB was reduced or interrupted as necessary in cases of local intolerance. Ointments were applied after irradiation or at least six hours beforehand. The treatment groups were comparable at the start of the study; the mean PASI scores of 17.4 for 49 patients in the calcitriol group and 16.6 for 53 patients in the control group were not significantly different. Mean global improvement scores increased throughout the study period, with the difference between treatments in favor of calcitriol plus UVB therapy being significant $(P<0.05)$ in all but one of the time points starting from week 1 . This clinical response was reflected in a highly significant difference $(P=0.005)$ in the mean percentage reduction in PASI score for patients treated with calcitriol plus UVB (65\%) compared with those receiving phototherapy alone (43\%). By study endpoint considerable or definite improvement or clearance of lesions was seen in $45 \%$ of patients treated with calcitriol plus UVB and in $20 \%$ of those receiving UVB alone. The mean cumulative UVB dose was $5,291 \mathrm{~J} \mathrm{~cm}^{-2}$ in the calcitriol and $8,668 \mathrm{~J} \mathrm{~cm}^{-2}$ in the excipient group, respectively. Thus, on average, a $34 \%$ lower dose of UVB was administered to calcitriol-treated patients than to those in the control group.

In another open intra-individual half-side manner study, ten patients with symmetrical plaque psoriasis were treated on one arm with calcitriol $3 \mu \mathrm{g} \mathrm{g}^{-1}$ ointment twice daily and on the other arm with dithranol ointment once daily. Additionally, these patients received narrow-band (311 nm) UVB phototherapy five times a week for at least four weeks. Both treatment modalities notably reduced the modified PASI score equally effectively. Three patients preferred calcitriol rather than dithranol and one patient vice versa when both quality of life and treatment acceptability were assessed. ${ }^{21}$

\section{Combinations with corticosteroids}

In a small, double-blind, randomized study patients applied either the potent topical corticosteroid betamethasone valerate $0.1 \%$ ointment in the morning and calcitriol $3 \mu \mathrm{g} \mathrm{g}^{-1}$ ointment in the evening $(\mathrm{n}=9)$, or betamethasone valerate ointment in both the morning and the evening $(n=10)$. Treatment lasted for six weeks. ${ }^{33}$ The combination therapy was at least as effective as betamethasone alone, as indicated by the three measures of efficacy that were assessed. At study endpoint $78 \%$ of patients using calcitriol/ betamethasone and $60 \%$ of those using betamethasone daily showed considerable improvement, or better, according to the mean global improvement score. Likewise, the global severity score at endpoint was 1 (slight) for $78 \%$ of the combination group and $50 \%$ of the betamethasone group. Finally, the mean PASI score for patients in the calcitriol/ betamethasone group decreased over the course of the study by $81 \%$ compared to $75 \%$ for betamethasone-treated patients. No statistical analyses were performed due to the small population size.

In another two-phase parallel-group study including 125 patients with chronic plaque type psoriasis either calcitriol $3 \mu \mathrm{g} \mathrm{g}^{-1}$ ointment or calcipotriol $50 \mu \mathrm{g} \mathrm{g}^{-1}$ 
ointment were administered in the morning and the topical corticosteroid clobetasol in the evening for two weeks, followed by monotherapy with either calcitriol or calcipotriol twice daily for another 10 weeks. At week 2 in both arms, more than $50 \%$ of the patients showed at least a marked improvement of their psoriasis and at week $12,79 \%$ in the calcitriol and $88 \%$ in the calcipotriol group, respectively. Least-square means analysis of the PASI indicated both regimens to be equivalent. There were no differences between the groups with regards to cutaneous safety or to incidence of adverse effects. ${ }^{23}$

\section{Open studies}

In three separate studies, calcitriol $3 \mu \mathrm{g} \mathrm{g}^{-1}$ ointment was used to treat moderate or severe lesions of chronic plaque psoriasis localized in sensitive skin areas. In an open, five-center study, treatment was applied for eight weeks by 11 male and 20 female patients with a history of psoriasis lasting from one to 757 months (mean 198 months) involving the face, hairline, or retroauricular areas. ${ }^{34}$ A good response to treatment, in terms of improvement in scaling, erythema, induration, and pruritus, was seen by week 4 which continued through to week 8 . The global improvement score indicated that at study endpoint, 74\% of patients were classified as definitely improved, or better. Another group of patients (12 males, 8 females) applied calcitriol $3 \mu \mathrm{g} \mathrm{g}^{-1}$ ointment for 6 weeks to psoriatic plaques on the face, hairline or retroauricular areas in a single-center study. ${ }^{17}$ The clinical outcome showed a similar pattern to the previous study, although a higher proportion of patients experienced clearing of their lesions. ${ }^{17}$ One patient discontinued therapy prematurely, after 33 days of twicedaily applications, due to complete clearance of lesions. In these two studies the cosmetic acceptability of treatment was judged as good by the vast majority of patients; only one patient considered the medication to be cosmetically unacceptable.

In another study 60 patients with mild-to-moderate plaque psoriasis involving sensitive areas were treated with calcitriol $3 \mu \mathrm{g} \mathrm{g}^{-1}$ ointment twice a day for 12 weeks. The clinical remission rate progressively increased throughout therapy from $11.6 \%$ at week 4 to $63.3 \%$ at week 12 . No serious adverse events and clinically relevant changes of calcium homeostasis were observed. Six patients withdrew after four weeks of treatment because of increasing pruritus. Another 12 patients noted mild skin irritation or pruritus which spontaneously disappeared in six patients during therapy. ${ }^{26}$
To assess the long-term efficacy and safety of calcitriol, an open-label multicenter study of 324 patients with mild-tomoderate plaque psoriasis were included. They received calcitriol $3 \mu \mathrm{g} \mathrm{g}^{-1}$ ointment twice a day for up to 52 weeks. 233 of the patients completed 26 weeks and 136 completed 52 weeks of treatment. Efficacy was demonstrated over the course of the treatment assessed with an investigator-rated severity score. This evaluation demonstrated no or minimal psoriasis symptoms in $11 \%$ of the treated patients after 12 weeks, in $22 \%$ after 24 weeks, in $37 \%$ after 36 weeks, and in $47 \%$ after 52 weeks. The psoriasis involved body surface area decreased from $16 \%$ at the beginning to $11 \%$ at the endpoints of the study. Serious adverse effects were reported in eight patients, all cases probably unrelated to the study medication (skin ulcer at distant site, joint disorder, two cases of metorrhagia, heart failure, hospitalization due to arteriosclerosis, breast cancer, and an infection following a dog bite). Ten patients showed transient hypercalcemia but none was clinically significant or led to discontinuation of the treatment. Furthermore, no clinically significant other laboratory test parameters occurred. The global assessment of improvement was rated by the patients. After 26 weeks $53 \%$ and after 52 weeks $64 \%$ of the patients reported at least a marked improvement of their psoriasis while $4 \%$ had discontinued the treatment due to lack of efficacy and $3 \%$ due to adverse effects. ${ }^{28}$

\section{Conclusions: Place of calcitriol in therapy of mild-to-moderate psoriasis}

Twice-daily calcitriol $3 \mu \mathrm{g} \mathrm{g}^{-1}$ ointment was demonstrated to be an effective and safe treatment for mild-to-moderate plaque psoriasis, ${ }^{20}$ including sensitive skin areas. ${ }^{17,26,34}$ The same is true for long-term treatment during and up to 52 weeks. $^{28}$

For chronic conditions such as psoriasis the availability of a range of therapies allows treatment to be adapted to patients' needs, clinical response, and tolerance. It is therefore important to determine the comparability and compatibility of different treatments and thereby to establish their place in the therapeutic armamentarium.

Investigation of the efficacy of twice-daily calcitriol $3 \mu \mathrm{g} \mathrm{g}^{-1}$ ointment shows it to be as effective as short contact dithranol, a well-established treatment for chronic plaque psoriasis. ${ }^{35}$ However, due to the much lower frequency of skin irritation and staining, calcitriol was associated with significantly better overall patient acceptability than dithranol cream. 
Topical betamethasone dipropinate, a corticosteroid, was demonstrated to be slightly more effective than calcitriol $3 \mu \mathrm{g} \mathrm{g}^{-1}$ ointment but a higher proportion of responders treated with the latter drug remained in remission over the next eight weeks. ${ }^{22}$ Comparative studies with topical calcipotriol $50 \mu \mathrm{g} \mathrm{g}^{-1}$, another vitamin D derivative, showed a tendency in favor of calcitriol $3 \mu \mathrm{g} \mathrm{g}^{-1}$ ointment regarding the efficacy, which furthermore had a significantly higher tolerance in respect of skin toxicity. ${ }^{24,25}$ No studies comparing topical calcitriol with topical retinoids have been published. In a comparison of topical tacrolimus $300 \mu \mathrm{g} \mathrm{g}^{-1}$, a calcineurin antagonist, not approved for treatment of psoriasis but for atopic eczema, with calcitriol $3 \mu \mathrm{g} \mathrm{g}^{-1}$ ointment for the treatment of psoriasis affecting sensitive skin areas like the face and the genitofemoral region, the former was demonstrated to be more effective and less irritating. ${ }^{27}$

The effective management of psoriasis frequently necessitates combining therapies in order to achieve optimum response while minimizing any side-effects. Several studies reviewed here show that calcitriol can be safely and effectively used in combination with either UVB phototherapy ${ }^{19,21}$ or a topical corticosteroid. ${ }^{23,33}$ In these studies the combination therapy proved to be at least as efficacious as the comparative therapy alone. In the case of phototherapy, the combination with calcitriol resulted in a significant reduction in the dose of UV radiation needed to produce an equivalent therapeutic response. A steroid-sparing effect was achieved by using calcitriol in the morning and betamethasone valerate in the evening; this combination was as effective as twice-daily betamethasone velerate. Therefore, in these studies benefit accrued from the combination with calcitriol; by allowing for a reduction in dose of the standard therapy, the risk of the adverse effects commonly associated with these therapies was decreased.

Many studies indicate that the systemic tolerance of calcitriol $3 \mu \mathrm{g} \mathrm{g}^{-1}$ is good. There was no evidence of clinically significant effects on calcium or phosphorus homeostasis either for the study populations as whole, or for individual patients. Although individual cases of asymptomatic hypercalcemia were recorded, they were transient and patients were able to continue with their treatment.

In conclusion, these studies provide ample evidence that calcitriol $3 \mu \mathrm{g} \mathrm{g}^{-1}$ ointment is an effective and safe treatment in the management and control of mild-to-moderate plaque psoriasis.

\section{Disclosure}

Dr Kowalzick has served as principal and clinical investigator and has been a speaker for Galderma Laboratories.

\section{References}

1. Langner A, Ashton P, van de Kerkhof PC, et al. A long-term multicentre assessment of the safety and tolerability of calcitriol ointment in the treatment of chronic plaque psoriasis. Br J Dermatol. 1996; 135:385-389.

2. Dubertret L, Wallach D, Souteyrand P, et al. Efficacy and safety of calcipotriol (MC 903) ointment in psoriasis vulgaris. A randomized, double-blind, right/left comparative, vehicle-controlled study. $J \mathrm{Am}$ Acad Dermatol. 1992;27:983-988.

3. Bourke JF, Mumford R. Whittaker P, et al. The effects of topical calcipotriol on systemic calcium homeostasis in patients with chronic plaque psoriasis. J Am Acad Dermatol. 1997;37:929-934.

4. Russell S, Young MJ. Hypercalcaemia during treatment of psoriasis with calcipotriol. Br J Dermatol. 1994;130:795-796.

5. Tham SN, Lun KC, Cheong WK. A comparative study of calcipotriol ointment and tar in chronic plaque psoriasis. Br J Dermatol. 1994;131:673-677.

6. Kragballe K, Barnes L, Hamberg KJ, et al. Calcipotriol cream with or without concurrent topical corticosteroid in psoriasis: tolerability and efficacy. Br J Dermatol. 1998;139:649-654.

7. Clemens TL, Adams JS, Horiuchi N, et al. Interaction of 1,25-dihydroxyvitamin- $\mathrm{D}_{3}$ with keratinocytes and fibroblasts from skin of normal subjects and a subject with vitamin-D-dependent rickets, type II. A model for study of the mode of action of 1,25-dihydroxyvitamin $\mathrm{D}_{3}$. J Clin Endocrinol Metab. 1983;56:824-830.

8. Hennings H, Michael D, Cheng C, Steinert P, Holbrook K, Yuspa SH. Calcium regulation of growth and differentiation of mouse epidermal cells in culture. Cell. 1980;19:245-254.

9. Bittiner B, Bleehen SS, MacNeil S. 1 alpha, $25(\mathrm{OH})_{2}$ vitamin $\mathrm{D}_{3}$ increases intracellular calcium in human keratinocytes. Br J Dermatol. 1991;124:230-235.

10. Hosomi J, Hosoi J, Abe E, et al. Regulation of terminal differentiation of cultured mouse epidermal cells by 1 alpha, 25-dihydroxyvitamin $\mathrm{D}_{3}$. Endocrinoloy. 1983;113:1950-1957.

11. Smith EL, Walworth NC, Holick MF. Effect of 1 alpha, 25-dihydroxyvitamin $\mathrm{D}_{3}$ on the morphologic and biochemical differentiation of cultured human epidermal keratinocytes grown in serum-free conditions. J Invest Dermatol. 1986;86:709-714.

12. Franssen ME, de Jongh GJ, van Erp PE, van de Kerkhof PC. A left/right comparison of twice daily calcipotriol ointment and calcitriol ointment in patients with psoriasis: the effect on keratinocyte subpopulations. Acta Derm Venereol. 2004;84:195-200.

13. Reichrath J, Perez A, Muller SM, et al. Topical calcitriol (1,25-dihydroxyvitamin $\mathrm{D}_{3}$ ) treatment of psoriasis: an immunohistological evaluation. Acta Derm Venereol. 1997;77:268-272.

14. van de Kerkhof PC. Reduction of epidermal abnormalities and inflammatory changes in psoriatic plaques during treatment with vitamin $\mathrm{D}_{3}$ analogs. J Invest Dermatol Symp Proc. 1996;1:78-81.

15. Rizova E, Corroller M. Topical calcitriol - studies on local tolerance and systemic safety. Br J Dermatol. 2001;144(S1):3-10.

16. van Haarten J, Lammers R, Boon C. Toxicokinetics and clinical kinetics of topically applied vitamin D analogues. J Dermatol. 1996;7:19-21.

17. Langner A, Stapor V, Ambroziak M. Vitamin $\mathrm{D}_{3}$ metabolites and analogues in the treatment of scalp psoriasis. J Dermatol Treat. 1998;9:41-45.

18. Hutchinson PE, Marks R, White J. The efficacy, safety and tolerance of topical calcitriol $3 \mu \mathrm{g} / \mathrm{g}$ ointment in the treatment of plaque psoriasis: a comparison with short-contact dithranol. Dermatology. 2000; 201:139-145.

19. Ring J, Kowalzick L, Christophers E, et al. Topical calcitriol $\left(1,25\right.$-dihydroxyvitamin $\left.\mathrm{D}_{3}\right)$ in the treatment of plaques psoriasis. $J$ Eur Acad Dermatol Venerol. 2000;14:S259-S260.

20. Lebwohl M, Menter A, Weiss J, et al. Calcitriol 3 microg/g ointment in the management of mild to moderate plaque type psoriasis: results from 2 placebo-controlled multicenter, randomized, double-blind, clinical studies. J Drugs Dermatol. 2007;6:428-435. 
21. Hofmann UB, Eggert AA, Bröcker EB, Goebeler M. Calcitriol vs dithranol in combination with narrow-band ultraviolet B $(311 \mathrm{~nm})$ in psoriasis. Br J Dermatol. 2003;148:779-783.

22. Camarasa JM, Ortonne JP, Dubertret L. Calcitriol shows greater persistence of treatment effect than betamethasone dipropionate in topical psoriasis therapy. Dermatolog Treat. 2003;14:8-13.

23. Lahfa M, Mrowietz U, Koenig M, Simon JC. Calcitriol ointment and clobetasol propionate cream: a new regimen for the treatment of plaque psoriasis. Eur J Dermatol. 2003;13:261-265.

24. Zhu X, Wang B, Zhao G, et al. An investigator masked comparison on the efficacy and safety of twice daily applications of calcitriol $3 \mathrm{microg} / \mathrm{g}$ ointment vs calcipotriol $50 \mathrm{microg} / \mathrm{g}$ ointment in subjects with mild or moderate chronic plaque-type psoriasis. Eur Acad Dermatol Venereol. 2007;21:466-472.

25. Ortonne JP, Humbert P, Nicolas JF, et al. Intra-individual comparison of the cutaneous safety and efficacy of calcitriol 3 microg $g(-1)$ ointment and calcipotriol 50 microg $\mathrm{g}(-1)$ ointment on chronic plaque psoriasis localized in facial, hairline, retroauricular or flexural areas. Br J Dermatol. 2003;148:326-333.

26. Carboni I, de Felice C, Bergamin A, Chimenti S. Topical use of calcitriol $3 \mathrm{microg} / \mathrm{g}$ ointment in the treatment of mild-to-moderate psoriasis: results from an open-label study. Eur Acad Dermatol Venereol. 2005;19,S3:11-13.

27. LiaoYH, Chiu HC, Tesng YS, Tsai TF. Comparison of cutaneous tolerance and efficacy of calcitriol 3 microg $\mathrm{g}(-1)$ ointment and tacrolimus $0.3 \mathrm{mg} g(-1)$ ointment in chronic plaque psoriasis involving facial or genitofemoral areas: a double-blind, randomized controlled study. Br J Dermatol. 2007;157:1005-1012.
28. Lebwohl M, Ortonne JP, Andres P, Briantais P. Calcitriol 3 microg/g ointment is safe and effective over 52 weeks for the treatment of mild to moderate plaque psoriasis. J Drugs Dermatol. 2007;6: 428-435.

29. Fredriksson T. Lassus A, Salde L. Reproducibility of clinical trials of topical glucocorticosteroids. Int J Dermatol. 1983;22:536-539.

30. Fredriksson T. Pettersson U. Severe psoriasis - oral therapy with a new retinoid. Dermatologica. 1978;157:238-244.

31. Finlay AY, Khan GK, Luscombe DK, et al. Validation of Sickness Impact Profile and Psoriasis Disability Index in psoriasis. Br J Dermatol. 1990;123:751-756.

32. Fortune DG, Main CJ, O 'Sullivan TM, et al. Quality of life in patients with psoriasis: the contribution of clinical variables and psoriasisspecific stress. Br J Dermatol. 1997;137:755-760.

33. Galderma Laboratories. A prospectively randomized double-blind, parallel group study of once daily betamethasone valerate $0,1 \%$ ointment (BV) in the morning and once daily calcitriol $3.0 \mu \mathrm{g} / \mathrm{g}$ in the evening versus twice daily BV in the treatment of patients suffering from chronic plaques psoriasis. Study Report No. 6, H141 901/MC. Lausanne, Switzerland: Galderma Laboratories; 1993. Data on file.

34. Galderma Laboratories. An open safety and tolerance study of calcitriol ointment $3.0 \mu \mathrm{g} / \mathrm{g}$ in the treatment of chronic plaque psoriasis including facial, hairline and retroauricular areas. Study Report No. 14, H141.414. Lausanne, Switzerland: Galderma Laboratories; 1994. Data on file.

35. van de Kerkhof PCM. Dithranol treatment for psoriasis: After 75 years, still going strong! Eur J Dermatol. 1991;1:79-89.
Clinical, Cosmetic and Investigational Dermatology

\section{Publish your work in this journal}

Clinical, Cosmetic and Investigational Dermatology is an international, peer-reviewed, open access, online journal that focuses on the latest clinical and experimental research in all aspects of skin disease and cosmetic interventions. All areas of dermatology will be covered; contributions will be welcomed from all clinicians and

\section{Dovepress}

basic science researchers globally. This journal is indexed on CAS The manuscript management system is completely online and includes a very quick and fair peer-review system, which is all easy to use. Visit http://www.dovepress.com/testimonials.php to read real quotes from published authors. 